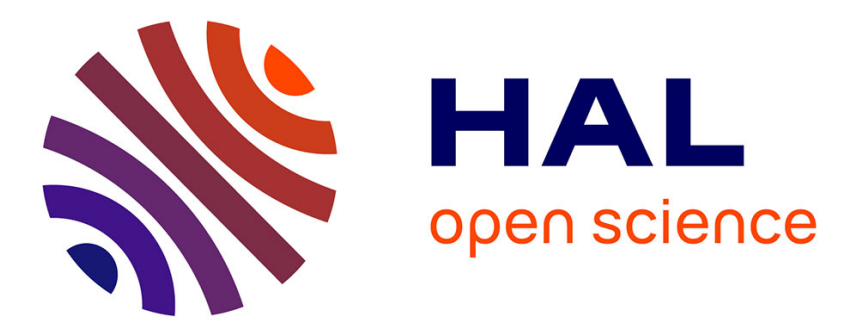

\title{
Minimal-excitation states for electron quantum optics using levitons
}

\author{
J. Dubois, T. Jullien, F. Portier, P. Roche, A. Cavanna, Y. Jin, W. \\ Wegscheider, P. Roulleau, D. C. Glattli
}

\section{- To cite this version:}

J. Dubois, T. Jullien, F. Portier, P. Roche, A. Cavanna, et al.. Minimal-excitation states for electron quantum optics using levitons. Nature, 2013, 502 (7473), pp.L659 - 663. 10.1038/nature12713 . cea-01481175

\section{HAL Id: cea-01481175 \\ https://hal-cea.archives-ouvertes.fr/cea-01481175}

Submitted on 2 Mar 2017

HAL is a multi-disciplinary open access archive for the deposit and dissemination of scientific research documents, whether they are published or not. The documents may come from teaching and research institutions in France or abroad, or from public or private research centers.
L'archive ouverte pluridisciplinaire $\mathbf{H A L}$, est destinée au dépôt et à la diffusion de documents scientifiques de niveau recherche, publiés ou non, émanant des établissements d'enseignement et de recherche français ou étrangers, des laboratoires publics ou privés. 


\section{Levitons : minimal excitations states of Fermions for electron quantum optics or cold atomic gases \\ T. Jullien $^{1}$, P. Roulleau ${ }^{1}$, J. Dubois ${ }^{1}$, F. Portier ${ }^{1}$, P. Roche ${ }^{1}$, Y. Jin ${ }^{2}$, A. Cavanna ${ }^{2}$ W. Wegscheider $^{3}$, and D. C. Glattli ${ }^{1}$ \\ ${ }^{1}$ Service de Physique de IŠEtat Condensé, IRAMIS/DSM (CNRS URA 2464), CEA Saclay, F- 91191 Gif-sur-Yvette, France \\ ${ }^{2}$ Laboratoire de Photonique et de Nanostructures (LPN), route de Nozay, 91460 Marcoussis, France) \\ ${ }^{3}$ Laboratory for Solid State Physics, ETH Zürich, CH-8093 Zürich, Switzerland \\ Presenting author's e-mail address: chisitian.glattli@cea.fr}

\section{Abstract:}

The on-demand generation of pure quantum excitations is a key issue to operate quantum systems. For Fermions this is particularly difficult as any perturbation affects all states below the Fermi energy resulting in a complex superposition of particles and holes excitations. However, it was predicted nearly twenty years ago [1] that a Lorentzian time dependent potential with quantized flux generates a minimal excitation with only one particle and no hole: a new quasiparticle called a leviton. Here, we report the on-demand generation of levitons in a conductor by applying voltage pulses on a contact [2]. Partitioning the excitations with an electronic beamsplitter generates a current noise used to measure their number. Minimal excitation states are observed for Lorentzian pulses while other pulse shapes show significant holes contribution. Further identification of levitons is provided in energy domain with shot noise spectroscopy and in time-domain with electronic Hong-Ou-Mandel noise correlation. The latter, obtained by colliding synchronized levitons on a beam-splitter, exemplifies the potential use of levitons for quantum information. Finally, we will present the first experimental tomography of an electron wavefunction in mesoscopic physics [3]. This is obtained by mixing levitons in the QPC beamsplitter with a weak fermion field prepared by applying a small ac sine-wave voltage on the opposite contact, following the theoretical work of ref.[4]. The anti-bunching of electrons and holes with the leviton provides a measure of the energy density matrix $\left\langle\psi^{+}(\varepsilon) \mid \psi\left(\varepsilon \pm k v_{0}\right)\right\rangle=\varphi(\varepsilon) \varphi\left(\varepsilon \pm k v_{0}\right)$ via excess shot noise measurements $\left(v_{0}\right.$ is the Leviton frequency injection, $\varphi(\varepsilon)$ its wave-function in energy representation). The present work demonstrates that, using levitons and linear electron quantum optics in ballistic conductors, let envisage flying qubit operation where the Fermi statistics is exploited to entangle synchronized electrons emitted by distinct sources. Compared with electron sources based on quantum dots, the generation of levitons does not require delicate nanolithography, considerably simplifying the circuitry for scalability. Finally, the principle of generation only exploiting fermionic properties can be applied to cold atomic gases $[5,6]$ where atomic levitons could be implemented.

[1] L. S. Levitov, H. Lee, and G. Lesovik, J. Math. Phys. 37, 4845 (1996) ; D. A. Ivanov, H. W. Lee, and L. S. Levitov, Phys. Rev B 56, 6839 (1997)

[2] J. Dubois, T. Jullien, P. Roulleau, F. Portier, P. Roche, Y. Jin, A. Cavanna W. Wegscheider, and D. C. Glattli, Minimal-excitation states for electron quantum optics using levitons, Nature 502, 659-663 (31 October 2013) 
[3] T. Jullien, P. Roulleau, B. Roche, A. Cavanna, Y. Jin and D. C. Glattli, tomography of an electron wave function, in preparation.

[4] C. Grenier et al 2011 New J. Phys. 13093007 (2011)

[5]J. P. Brantut et al, Conduction of Ultracold Fermions Through a Mesoscopic Channel, Science 337, 1069-1071 (2012)

[6] J. H. Thywissen, R. M. Westervelt, M. Prentiss, Quantum point contacts for neutral atoms, Phys. Rev. Lett. 83, $3762(1999)$

Acknowledgement: the ERC Adg \#228273 MeQuaNo is acknowledged.

\section{Figures}
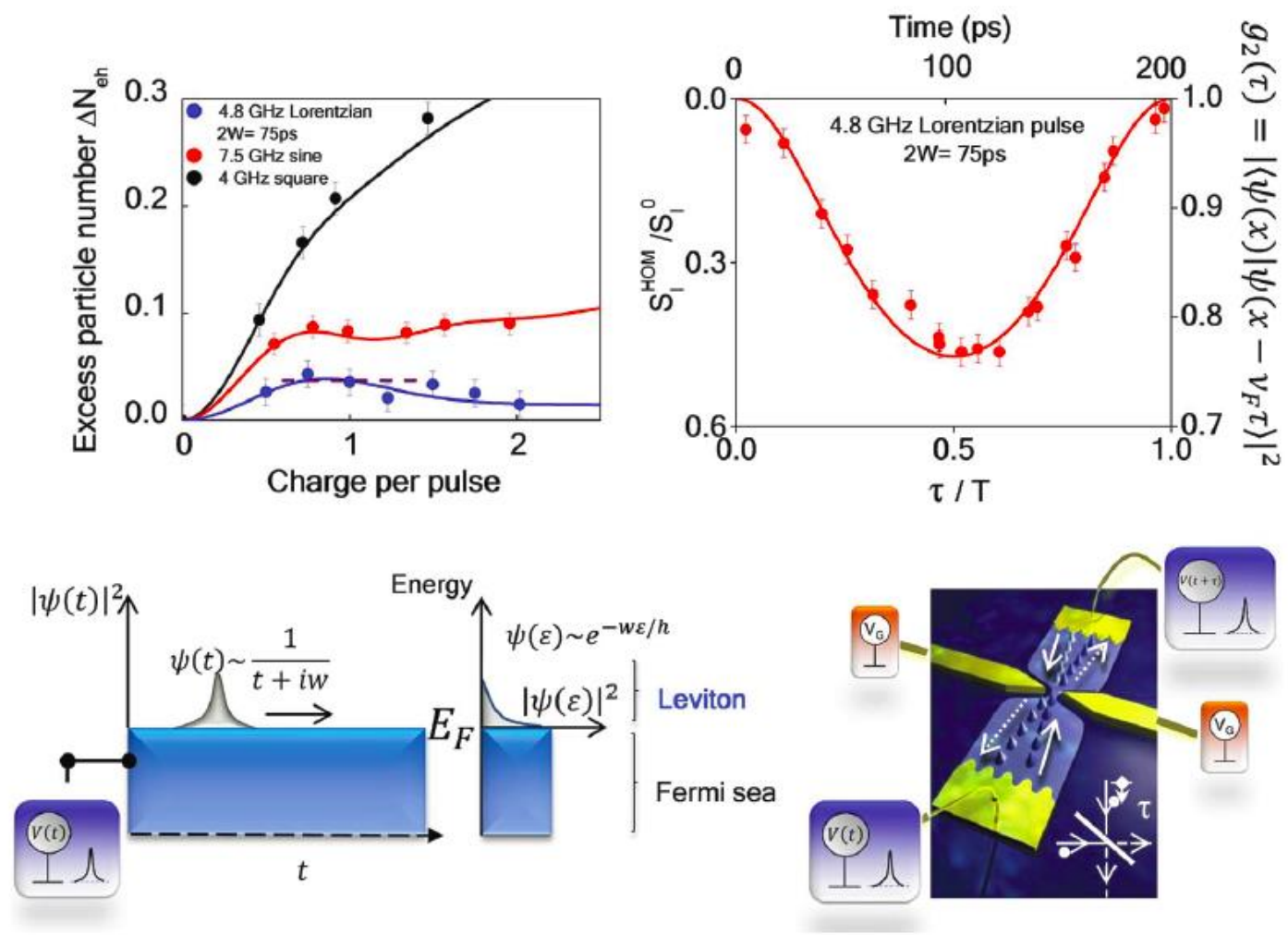

Figure : Upper left: partition noise of periodic charge pulses versus the charge carried per period observed for the application of various pulse shapes. The Lorentzian voltage pulse generates the minimal excitation. The residual noise observed for $\mathrm{q}=1$ and 2 is entirely due to thermal excitations. Lower left: schematics of the leviton wavefunction in time and energy domain. Upper right: electronic Hong Ou Mandel noise correlation provides a direct measure of the convolution of the Leviton wave-function in the time-domain. The principle is shown in the lower right figure. $\tau$ is the delay between the injection of synchronized indistinguishable levitons injected at opposite contact of the QPC-beam-splitter. (all figures from J. Dubois et al, Nature 2013, ref. [2]). 